\title{
Dynamic Complexity in a Prey-Predator Model with State-Dependent Impulsive Control Strategy
}

\author{
Chuanjun Dai $\mathbb{D}^{1,2}$ \\ ${ }^{1}$ Zhejiang Provincial Key Laboratory for Water Environment and Marine Biological Resources Protection, Wenzhou University, \\ Wenzhou, Zhejiang 325035, China \\ ${ }^{2}$ School of Life and Environmental Science, Wenzhou University, Wenzhou, Zhejiang 325035, China
}

Correspondence should be addressed to Chuanjun Dai; chuanjdai@wzu.edu.cn

Received 3 March 2020; Revised 25 May 2020; Accepted 16 June 2020; Published 6 July 2020

Guest Editor: Raúl Villafuerte-Segura

Copyright (C) 2020 Chuanjun Dai. This is an open access article distributed under the Creative Commons Attribution License, which permits unrestricted use, distribution, and reproduction in any medium, provided the original work is properly cited.

In this paper, an ecological model described by a couple of state-dependent impulsive equations is studied analytically and numerically. The theoretical analysis suggests that there exists a semitrivial periodic solution under some conditions and it is globally orbitally asymptotically stable. Furthermore, using the successor function, we study the existence, uniqueness, and stability of order-1 periodic solution, and the boundedness of solution is also presented. The relationship between order- $k$ successor function and order- $k$ periodic solution is discussed as well, thereby giving the existence condition of an order-3 periodic solution. In addition, a series of numerical simulations are carried out, which not only support the theoretical results but also show the complex dynamics in the model further, for example, the coexistence of multiple periodic solutions, chaos, and perioddoubling bifurcation.

\section{Introduction}

Since mathematical models represented by differential equations were introduced into biological and ecological systems, it has been proved that they are very useful tools to deal with practical problems such as emerging disease [1] and population dynamics between plankton and nutrient [2-4], which also promote the development of the theory of differential equation further. Especially, in the process, there is a significant development in impulsive differential equations [5-9] because many reality systems exhibit the abrupt jumps phenomena in population sizes during the evolution processes. Additionally, a theoretical method is urgently needed to provide a guide for the management of some practical problems such as pest outbreaks, phytoplankton blooms, vaccination, and so on. Accordingly, impulsive differential equation theory may prove to be one of the most potential theories to strategize about the approach of management for these problems, as impulsive differential equation can model abrupt jump behaviors induced by management, for example, some management occurs at fixed time, which can be modeled using the models with impulses at fixed times [10-13].

State-dependent impulsive differential equation, as a branch of impulsive differential equation, has the advantage to model the control behaviors depending on the population state. In recent years, the studies on state-dependent impulsive differential equation have been paid increasing attention [13-16]. Many studies devote to the properties of periodic solutions [17-20], including the existence, stability and periodicity, etc. It has been confirmed that the Poincaré map is a very useful tool to prove the existence of periodic solution in state-dependent impulsive differential equations [21-23]. In addition, Simeonov and Bainov [24] gave analogue of Poincaré criterion in ref. [25], which contributes to the stability of the periodic solution. Based on the Poincaré map, it can be proved theoretically that the positive periodic solution can bifurcate from the trivial periodic solution through a transcritical bifurcation [25]. Particularly, Chen [26] introduced the successor function a in semicontinuous dynamical system described by state-dependent impulsive differential equation to prove the existence of periodic 
solution. Using the successor function, Dai et al. [27] studied homoclinic bifurcation in a semicontinuous dynamical system [28]. The successor function has been extensively employed to investigate the existence of periodic solution and even stability and bifurcation in state-dependent impulsive differential equation [28-38]. These studies enrich the theory of state-dependent impulsive differential equation further.

In this paper, we will present a prey-predator two-species model with state-dependent impulsive control strategy to study the dynamics induced by impulsive control. Firstly, a model describing population dynamics of prey and predator is given below.

$$
\left\{\begin{array}{l}
\frac{\mathrm{d} x}{\mathrm{~d} t}=r x\left(1-\frac{x}{K}\right)-\frac{b x y}{a+x} \\
\frac{\mathrm{d} y}{\mathrm{~d} t}=\frac{\alpha b x y}{a+x}-m y
\end{array}\right.
$$

where $x$ and $y$ denote the densities of prey and predator, respectively; $r$ is the intrinsic growth rate of the prey; $K$ is the carrying capacity of the prey; $b$ is the maximum growth rate; $a$ represents the half-saturation concentration; $\alpha$ is the assimilation efficiency of predator; and $m$ is the specific mortality rate of predator. Here, we employ a Holling II functional response to present the interaction between prey and predator because some results suggest that the population dynamic following Holling II may exist in reality $[2,3,39,40]$.

For some species, when their densities are beyond a certain value, some seriously negative effects will emerge, for instance, algal bloom. For this reason, their densities should be kept below the critical value by harvest or/and released natural enemy. Obviously, the occurrence of control will rely on the density of the species. Here, under the assumption that the interaction between prey $x$ and predator $y$ follows model (1), we investigate the dynamics of impulsive control and control strategies for prey $x$ using state-dependent impulsive differential equation, and the model can be described as

$$
\left\{\begin{array}{l}
\frac{\mathrm{d} x}{\mathrm{~d} t}=r x\left(1-\frac{x}{K}\right)-\frac{b x y}{a+x} \\
\frac{\mathrm{d} y}{\mathrm{~d} t}=\frac{\alpha b x y}{a+x}-m y \\
\left.\begin{array}{rl}
\Delta x & =-p x \\
\Delta y & =-q y+\tau
\end{array}\right\} \quad x=h
\end{array} \quad \begin{array}{l}
x<h \\
x=h
\end{array}\right.
$$

where $h>0$ denotes the critical value below which the density of prey $x$ should be kept. $0<p<1$ is the harvest rate of prey $x$; $\tau \geq 0$ is the releasing amounts of predator $y$; and $0<q<1$ represents the harvest rate of predator $y$ or the level of negative effect caused by the harvest of prey $x$ on predator $y$.
The rest of the paper is organized as follows. In Section 2, some notations, basic definitions, and lemmas are given. In Section 3, a semitrivial solution and its stability are studied; we also study the existence, uniqueness, and stability of an order-1 periodic solution; moreover, the boundedness of solution and some propositions of periodic solution are discussed as well. Whereafter, a series of numerical results are carried out to study the dynamics of model (2) further in Section 4. Finally, the paper ends with the conclusion in Section 5 .

\section{Preliminaries}

In model (1), there exist three equilibria, $E_{0}=(0,0)$, $E_{1}=(K, 0)$, and $E_{*}=\left(x_{*}, y^{*}\right)$, where $x_{*}=m a /(\alpha b-m)$ and $y^{*}=r\left(K-x_{*}\right)\left(a+x_{*}\right) /(b K)$. When the condition $\max (0, \alpha b(K-a) /(K+a))<m<K \alpha b /(a+K)$ holds, $E_{*}$ is a stable positive focus or node. We assume that the following condition holds throughout the paper: $(H)$ $0<m<K \alpha b /(a+K)$.

The vertical isocline and the horizontal isocline can be defined as follows:

$$
\begin{aligned}
& L_{1}: y=\frac{r(K-x)(a+x)}{b K}=f(x), \\
& L_{2}: x=\frac{m a}{\alpha b-m} .
\end{aligned}
$$

Furthermore, let $N=\{(x, y) \mid x=(1-p) h, y \geq 0\}$ be the phase set, and $M=\{(x, y) \mid x=h, y \geq 0\}$ denotes the impulsive set. Under condition $(H)$, we assume that $L_{1}$ intersects with the phase set $N$ at point $O\left(x_{O}, y_{O}\right)$ and the impulsive set $M$ at point $H\left(x_{H}, y_{H}\right)$ in the first quadrant when $h<x_{*}$. We define $\Pi=\{(x, y) \mid(1-p) h \leq$ $x \leq h, 0 \leq y \leq f(x)\}$ and $\Omega=\{(x, y) \mid 0 \leq x \leq h, y \geq 0\}$.

Let $z(t)=(x(t), y(t))$ be any solution of model (2); then, we define the positive trajectory initializing at point $z_{0}=z\left(t_{0}\right) \in R_{+}^{2}=\{(x, y) \mid x \geq 0, y \geq 0\}$ for $t \geq t_{0}$ as $\pi\left(t ; z_{0}, t_{0}\right)=\left\{z(t) \mid z(t) \in R_{+}^{2}, t \geq t_{0} \geq 0, z\left(t_{0}\right)=z_{0}\right\}$.

In this paper, some notions are always mentioned, including phased point, impulsive point, and successor point. From works in $[20,23,26]$, we state the following definition.

Definition 1. Take the point $A_{0} \in N$; set $\pi\left(t ; A_{0}, t_{0}\right)$ as the trajectory initializing at point $A_{0}$ in model (2). If there exists $t_{k}>t_{0}$ such that the trajectory $\pi\left(t ; A_{0}, t_{0}\right)$ intersects the impulsive set $M$ at $A_{k}=\left(h, y_{k}\right)$ when $t=t_{k}(k=1,2,3, \ldots)$, then a point $A_{k}^{+}$exists in the phase set $N$, where point $A_{k}^{+}=\left((1-p) h, y_{k}^{+}=(1-q) y_{k}+\tau\right)$. The point $A_{k}^{+}$is called the phased point of point $A_{k}$, and the point $A_{k}$ is called the impulsive point of point $A_{k}^{+}$. The point $A_{k}^{+}$is called the order$k$ successor point of point $A_{0}$, and the vector $\overrightarrow{A_{k} A_{k}^{+}}$is called an impulsive line.

Definition 2. Set point $E=N \cap\{(x, y) \mid x \geq 0, y=0\}$; then, we define a number axis $L$ in the phase $N$, and set point $E$ as the origin of the number axis $L . \forall A_{0} \in N$, set the direction of 
vector $\overrightarrow{E A_{0}}$ as the positive direction of number axis $L$, and set $a_{0}=\left|\overrightarrow{E A_{0}}\right|$ as the coordinate of point $A_{0}$ in the number axis $L$. Then, a map $F_{k}: R_{+} \longrightarrow R(k=1,2,3, \ldots)$ can be defined as $F_{k}\left(a_{0}\right)=a_{k}-a_{0}$, where $a_{k}=\left|\overrightarrow{E A_{k}^{+}}\right|$and $A_{k}^{+}$is the order- $k$ successor point of point $A_{0}$. The map $F_{k}$ is called the order- $k$ successor function, that is, $F_{k}\left(A_{0}\right)=y_{A_{k}^{+}}-y_{A_{0}}$.

Definition 3. Take a point $S$ in the phase set $N$; then, $\pi\left(t ; S, t_{0}\right)$ is a solution of model (2). If there exists a positive integer $k \geq 1$ such that $k$ is the smallest integer for $F_{k}(S)=0$, then the solution $\pi\left(t ; S, t_{0}\right)$ is called order- $k$ periodic solution.

Lemma 1 (see [26]). The successor function $F_{k}(S)$ is continuous, where the point $S \in N$.

Obviously, we can obtain the following lemma by Lemma 1.

Lemma 2. If there exist two points $S_{1}=\left((1-p) h, y_{S_{1}}\right)$ and $S_{2}=\left((1-p) h, y_{S_{2}}\right)$ in model (2) and $\left(y_{S_{1}}-y_{O}\right)$ $\left(y_{S_{2}}-y_{O}\right) \geq 0$ such that $F_{1}\left(S_{1}\right) F_{1}\left(S_{2}\right)<0$, then model (2) has an order-1 periodic solution.

For the point $O$, we always assume the point $O_{k}^{+}=((1-$ $\left.p) h, y_{O_{k}^{+}}\right)$is the order- $k$ successor point of the point $O$, and the point $O_{k}=\left(h, y_{O_{k}}\right)$ is the impulsive point of point $O_{k}^{+}$, where $k=1,2,3, \ldots$..

\section{Main Results}

When the values of parameters in model (1) are given, the dynamics of model (2) only depend on the parameters $h, p$, $q$, and $\tau$. In this paper, we are interested in the effect of impulsive control on dynamics of model (2). Hence, we will investigate the dynamical behaviors of model (2) under the condition that the parameters of model (1) are fixed.

For points $O$ and $H$, it is obvious that

(1) $y_{O}>y_{H}$ if $(1-p / 2) h>K-a / 2$.

(2) $y_{O}<y_{H}$ if $(1-p / 2) h<K-a / 2$ and $K>a$.

In this section, we just show the proof with respect to case $(K-a) / 2<(1-p) h<(1-p / 2) h$ in the figure of illustration. The proof corresponding to other cases is similar, which has been omitted.

By model (1), it is obvious that $h<x_{*}$ implies $\mathrm{d} y / \mathrm{d} t<0$. Additionally, $y\left(t^{+}\right)<y(t)$ always holds when $q \in(0,1)$.

In model (2) with $\tau=0$, it is obvious that the following semitrivial periodic solution exists for $t \in((n-1) T, n T](n=1,2,3, \ldots)$ when the predator $y=0$,

$$
\left\{\begin{array}{l}
\xi(t)=\frac{(1-p) h e^{r(t-(n-1) T)} K}{(1-p) h e^{r(t-(n-1) T)}+(K-(1-p) h)}, \\
\eta(t)=0,
\end{array}\right.
$$

where the period $T=(1 / r) \ln (K-(1-p) h /(1-p)$ $(K-h))$. Set $\Gamma=(\xi(t), \eta(t))$. Then, we can get the following theorem.
Theorem 1. Under condition $(H)$, if $h<x_{*}$ and $\tau=0$, then semitrivial periodic solution $\Gamma$ is globally orbitally asymptotically stable in model (2) for any $q \in(0,1)$ and $p \in(0,1)$.

Proof. For $\tau=0$, solutions of model (2) will enter the space $\Pi$ eventually. Hence, we study the stability of the semitrivial periodic solution in this space.

For any $\varepsilon>0$, set $N_{\varepsilon}=((1-p) h, \varepsilon)$ and $M_{\varepsilon}=(h, \varepsilon)$. Then, we assume that the trajectory $\pi\left(t ; N_{\varepsilon}, t_{0}\right)$ intersects with impulsive set $M$ at point $P=\left(h, y_{P}\right)$, and $y_{P}<y_{N_{\varepsilon}}=\varepsilon$ under condition $(H)$. Set $\delta=\varepsilon$; then, for any point $G=$ $\left((1-p) h, y_{G}\right)$ where $0<y_{G}<\delta=y_{N_{\varepsilon}}$, we have $y_{G_{1}^{+}}<y_{P}$ under condition $(H)$ because $h<x_{*}$ and $q \in(0,1)$ (see Figure 1(a)), which suggests that the distance

$$
d=d\left(\pi\left(t ; G, t_{0}\right), \Gamma\right)=\inf _{P_{0} \in \Gamma}\left|\pi\left(t ; G, t_{0}\right)-P_{0}\right|<\varepsilon
$$

holds for all $t>t_{0}$, where $\Gamma=(\xi(t), \eta(t))$. Hence, according to the definition of orbital stability in ref. [40], the semitrivial periodic solution $\Gamma$ is orbitally stable.

For any $G_{0} \in N$, take a sequence of number $\left\{t_{k}\right\}(k=1,2,3, \ldots)$, where $\quad 0<t_{k}<t_{k+1} \quad$ and $\lim _{k \longrightarrow+\infty} t_{k}=+\infty$, such that $\pi\left(t_{k} ; G_{0}, t_{0}\right)=$ $\left((1-p) h, y_{k}\right) \in N$. Then, the sequence of number $\left\{y_{k}\right\}$ is a strictly decreasing positive sequence because $h<x_{*}$ and $q \in(0,1)$. Hence, there exists a $y_{*} \geq 0$, such that $\lim _{k \longrightarrow+\infty} y_{k}=y_{*} \geq 0$, but $y_{k} \geq y_{*}$. Set $Q_{*}=\left((1-p) h, y_{*}\right)$, and let point $Q_{*}$ be the order-1 successor point of point $Q_{\#}=$ $\left((1-p) h, y_{\#}\right)$ if $y_{*}>0$, where $y_{\#}>y_{*}$. Then, for any point $Q \in \overline{Q_{*} Q_{\#}}$ (i.e., $y_{Q_{*}}<y_{Q}<y_{Q_{\#}}$ ), its order-1 successor point is below the point $Q_{*}$ (see Figure $1(\mathrm{~b})$ ), that is, $y_{Q^{+}} \leq y_{*}$.

Because $\lim _{k \longrightarrow+\infty} y_{k}=y_{*}$, there exists a positive integer $\Theta_{1}$ such that $y_{Q_{*}}<y_{k}<y_{Q_{\#}}$ for $k>\Theta_{1}$. According to above analysis, there exists a positive integer $\Theta_{2}>\Theta_{1}$ such that $y_{k}<y_{*}$ when $k>\Theta_{2}$, which contradicts with $y_{k} \geq y_{*}$. Hence, $\lim _{k \longrightarrow+\infty} y_{k}=y_{*}=0$, which means that the semitrivial periodic solution $\Gamma$ is orbitally attractive. So, the semitrivial periodic solution $\Gamma$ is orbitally asymptotically stable. Since the attraction domain is $\Omega$, the semitrivial periodic solution $\Gamma$ is globally orbitally asymptotically stable.

This completes the proof.

Suppose that $M$ is a positive constant, and then we have the following theorem for any $M \in(0,+\infty)$.

Theorem 2. Under condition $(H)$, if $h<x_{*}$ and $\tau \in(0, M]$, then there exists a unique order-1 periodic solution in model (2) for any $q \in(0,1)$ and $p \in(0,1)$.

Proof. For any $\tau>0$, Figure 2(a) displays the relation between point $O$ and its order- 1 successor point $O_{1}^{+}$under condition $(H)$, where $h<x_{*}$. Obviously, $\overline{O O_{1} O}$ is an order-1 periodic solution if $y_{O}=y_{O_{1}^{+}}$. If $y_{O}<y_{O_{1}^{+}}$, we have $F_{1}(O)=$ $y_{O_{1}^{+}}-y_{O}>0$ and $F_{1}\left(O_{1}^{+}\right)=y_{O_{2}^{+}}-y_{O_{1}^{+}}<0$, where $O_{2}^{+}$is the successor point of point $\mathrm{O}_{1}^{+}$. According to Lemmas 1 and 2, there exists an order-1 periodic solution in model (2).

If $y_{O}>y_{O^{+}}$, we take a point $R=((1-p) h, \tau)$ in phase set $N$. Then, let the point $R_{1}^{+}$be the order- 1 successor point of point $R$ and point $R_{1}$ be the impulsive point of point $R_{1}^{+}$, and $y_{R_{1}^{+}}=(1-q) y_{R_{1}}+\tau>\tau=y_{R}$ (see Figure $\left.2(\mathrm{a})\right)$, so $F_{1}(R)>0$. 


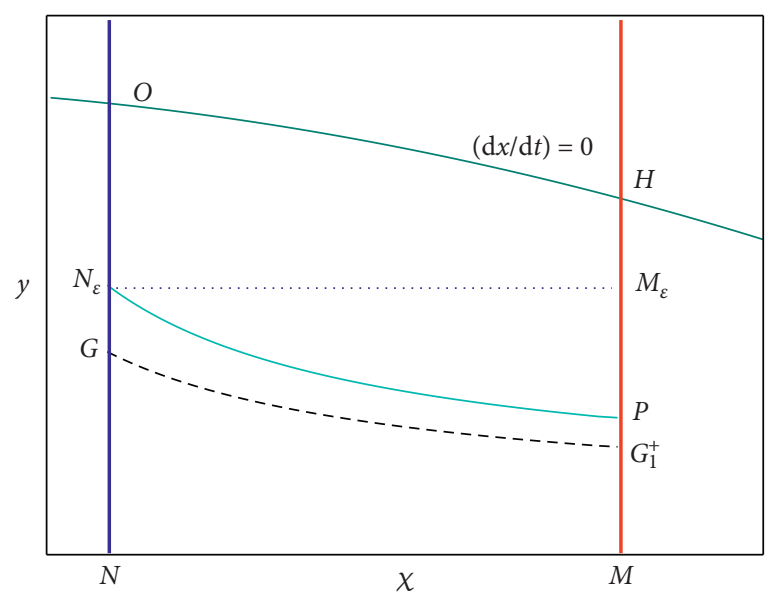

(a)

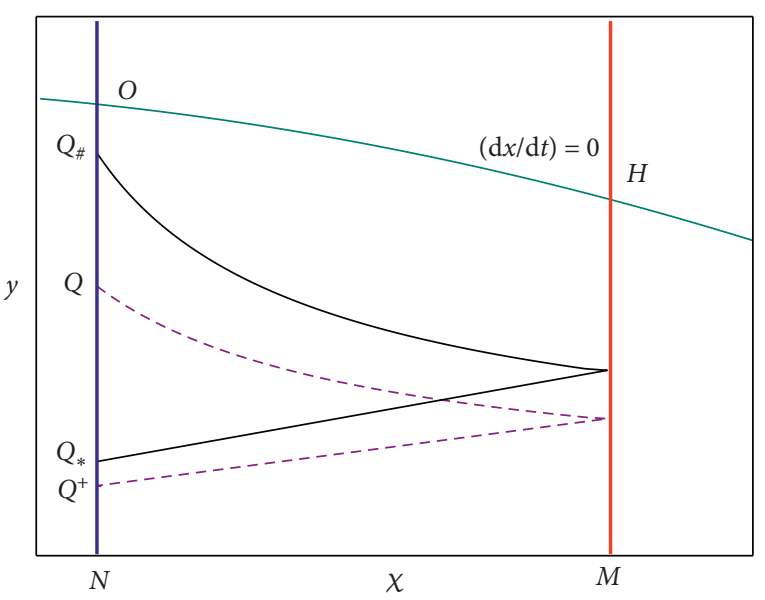

(b)

FIGURE 1: Illustration corresponding to the orbital (a) stability and (b) attractability of semitrivial periodic solution in model (2).

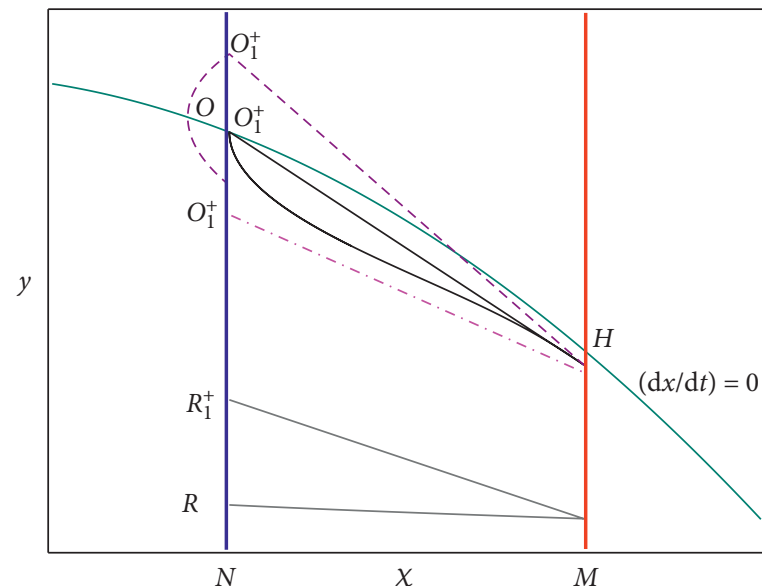

(a)

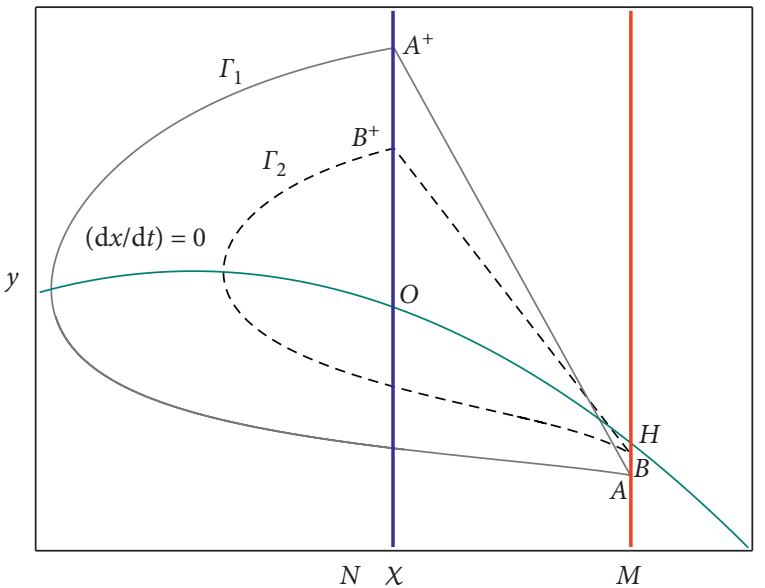

(b)

Figure 2: Illustration with respect to (a) existence and (b) uniqueness of the order-1 periodic solution in model (2).

By $F_{1}(O)=y_{O_{1}^{+}}-y_{O}<0$, there exists an order-1 periodic solution in model (2) using Lemmas 1 and 2.

Hence, there exists an order-1 periodic solution for any $q \in(0,1)$ and $p \in(0,1)$ when $h<x_{*}$ and $\tau>0$.

Suppose there are two order-1 periodic solutions in model (2), which are $\Gamma_{1}$ and $\Gamma_{2}$, respectively. Let point $A^{+}=$ $\left((1-p) h, y_{A^{+}}\right)$and $A=\left(h, y_{A}\right)$ be the phase point and impulsive point of $\Gamma_{1}$, respectively, and point $B^{+}=((1-$ $\left.p) h, y_{B^{+}}\right)$and $B=\left(h, y_{B}\right)$ be the phase point and impulsive point of $\Gamma_{2}$, respectively. Without loss of generality, set $y_{A^{+}}>y_{B^{+}}$. Then, the relation among $A^{+}, B^{+}$, and $O$ is (i) $B^{+} \in \overline{O A^{+}}\left(y_{O}<y_{B^{+}}<y_{A^{+}}\right)$; (ii) $A^{+} \in \overline{B^{+} O}\left(y_{B^{+}}<y_{A^{+}}<y_{O}\right)$; and (iii) $O \in \bar{B}^{+} A^{+}\left(y_{B^{+}}<y_{O}<y_{A^{+}}\right)$.

Figure 2(b) shows case (i); it is obvious that $\Gamma_{1}$ and $\Gamma_{2}$ cannot coexist by disjoint property of trajectory of model (1). Hence, the order-1 periodic solution is unique for this case.

For case (ii), because $A^{+} \in \overline{B^{+} O}$, we only need to focus on the region $\Pi$. We rewrite the model (1) in the region $\Pi$ as the following initial-value problem:

$$
\left\{\begin{array}{l}
\frac{\mathrm{d} y}{\mathrm{~d} x}=\frac{g(x, y)}{f(x, y)} \triangleq \Psi(x, y), \\
y\left(x_{0}\right)=y_{0}, \quad(x, y) \in \Pi,
\end{array}\right.
$$

where $f(x, y)=r x(1-x / K)-b x y / a+x, g(x, y)=\alpha b x y /$ $a+x-m y$.

Obviously, the function $\Psi(x, y)$ is continuously differentiable and monotone decreasing in $y$ on the space $\Pi$. Then, the solution of model (6) is equivalent to the following integral equation:

$$
y\left(x, y_{0}\right)=y_{0}+\int_{x_{0}}^{x} \Psi\left(s, y\left(s, y_{0}\right)\right) \mathrm{d} s .
$$

By equation (7), set

$$
y_{i}(x)=y\left(x, y_{i^{+}}\right)=y_{i^{+}}+\int_{(1-p) h}^{x} \Psi\left(s, y_{i}(s)\right) \mathrm{d} s,
$$


where $i=A, B$; then, $y_{A}=y_{A}(h)$ and $y_{B}=y_{B}(h)$. Because $y_{A^{+}}>y_{B^{+}}$, we have $y_{A}(x)>y_{B}(x)$ on the space $\Pi$ by the disjoint property of trajectory of model (1). Hence,

$$
\begin{aligned}
y_{A}-y_{B}= & y_{A}(h)-y_{B}(h) \\
= & y_{A^{+}}+\int_{(1-p) h}^{h} \Psi\left(x, y_{A}(x)\right) \mathrm{d} x-y_{B^{+}} \\
& -\int_{(1-p) h}^{h} \Psi\left(x, y_{B}(x)\right) \mathrm{d} x \\
& <y_{A^{+}}-y_{B^{+}} .
\end{aligned}
$$

On the other hand, because both $\Gamma_{1}$ and $\Gamma_{2}$ are order-1 periodic solutions, we have $y_{A^{+}}-y_{B^{+}}=(1-q)$ $\left(y_{A}-y_{B}\right)<\left(y_{A}-y_{B}\right)$ by model (2), which is a contradiction with inequality (9). Hence, the order-1 periodic solution is unique for this case.

For case (iii), the proof is similar with case (ii), so it is omitted.

Hence, there exists a unique order-1 periodic solution in model (2) for any $q \in(0,1)$ and $p \in(0,1)$ when $h<x_{*}$ and $\tau>0$.

This completes the proof.

From Theorem 2, we can find that the existence and stability of order-1 periodic solution depend on parameter $\tau$. Suppose point $((1-p) h, y(\tau))$ is the phase point of the order-1 periodic solution, then we can get the following proposition.

Proposition 1. Under condition $(H)$, when $h<x_{*}$ and $\tau>0$, $y=y(\tau)$ is a monotonously increasing function with respect to $\tau$.

Proof. Let $\theta$ be the angle between the impulsive line and the $x$-axis. Then, $\tan \theta=-q y+\tau /-p h$, so $\mathrm{d} \theta / \mathrm{d} \tau=(\mathrm{d} / \mathrm{d} \tau) \tan ^{-1}$ $(-q y+\tau /-p h)<0$. Hence, $\theta(\tau)$ is a monotonously decreasing function in $\tau$. Given an order-1 periodic solution $\Gamma_{*}$, let points $U=\left(x_{U}, y_{U}\right)$ and $W=\left(x_{W}, y_{W}\right)$ be the phased point and impulsive point of $\Gamma_{*}$, respectively; then, $\tau_{1}=$ $y_{U}-(1-q) y_{W}$ guarantees the existence of the order-1 periodic solution $\Gamma_{*}$.

For any $\varepsilon>0$, when $\tau=\tau_{1}+\varepsilon$, the order-1 periodic solution $\Gamma_{*}$ will disappear. Let point $U^{+}=\left(x_{U^{+}}, y_{U^{+}}\right)$be the order-1 successor point of point $U$; then, $y_{U^{+}}>y_{U}$ because $\theta(\tau)$ is a monotonously decreasing function. In addition, $F_{1}(U)>0$ and $F_{1}\left(U^{+}\right)<0$. Therefore, there exists an order-1 periodic solution whose phase point belongs to $\overline{U^{+}}$. By the uniqueness of order- 1 periodic solution, $y=y(\tau)$ is a monotonously increasing function in $\tau$.

This completes the proof.

By Theorem 2, we know that the trajectory $\overline{O O_{1} O}$ is an order-1 periodic solution of model (2) when $\tau=y_{O}-(1-q) y_{O_{1}} \triangleq \tau_{c}$. Then, we can get the following theorem.

Theorem 3. Under condition $(H)$, if $h<x_{*}$, then the order-1 periodic solution is globally orbitally asymptotically stable in model (2) for any $q \in(0,1)$ and $p \in(0,1)$ when $\tau \in\left(0, \tau_{c}\right)$.
Proof. When $\tau \in\left(0, \tau_{c}\right)$, the order-1 periodic solution of model (2) must belong to space $\Pi$ under condition $(H) . \Gamma^{+}$ denotes an order-1 periodic solution of model (2), whose phased point and impulsive point are point $C=\left(x_{C}, y_{C}\right)$ and point $D=\left(x_{D}, y_{D}\right)$, respectively. In addition, the solutions of model (2) will enter the space $\Pi$ eventually when $\tau \in\left(0, \tau_{c}\right)$. Hence, we only need to study the stability of order-1 periodic solution on the space $\Pi$.

For any $\varepsilon>0$, we assume that the trajectory $\pi\left(t ; N_{\varepsilon 1}, t_{0}\right)$ meets impulsive set $M$ at point $P_{1}=\left(x_{P_{1}}, y_{P_{1}}\right)$, where $N_{\varepsilon 1}=$ $\left((1-p) h, \varepsilon+y_{C}\right)$ (see Figure 3(a)); then, $y_{P_{1}}<y_{N_{\varepsilon 1}}=y_{C}+\varepsilon$. Take $\delta=\varepsilon$; for any point $G_{1}=\left(x_{G_{1}}, y_{G_{1}}\right)$, where $x_{G_{1}}=(1-$ $p) h$ and $y_{C}<y_{G_{1}}<y_{C}+\delta=y_{N_{\varepsilon 1}}$, we assume that the trajectory $\pi\left(t ; G_{1}, t_{0}\right)$ intersects with impulsive set at point $G_{2}=\left(h, y_{G_{2}}\right)$; then, $y_{G_{1}}>(1-q) y_{G_{2}}+\tau$; otherwise, another order-1 periodic solution exists above $\Gamma^{+}$, which contradicts with the uniqueness of order-1 periodic solution. Hence, the distance

$$
d=d\left(\pi\left(t ; G_{1}, t_{0}\right), \Gamma^{+}\right)=\inf _{P_{0} \in \Gamma^{+}}\left|\pi\left(t ; G_{1}, t_{0}\right)-P_{0}\right|<\varepsilon
$$

holds for all $t>t_{0}$ according to equation (9).

On the other hand, for any $0<\varepsilon<y_{C}$, given $\delta=\varepsilon$, when $y_{C}-\delta<y_{G_{1}}<y_{C}$, we still have

$$
d=d\left(\pi\left(t ; G_{1}, t_{0}\right), \Gamma^{+}\right)=\inf _{P_{0} \in \Gamma^{+}}\left|\pi\left(t ; G_{1}, t_{0}\right)-P_{0}\right|<\varepsilon,
$$

using the same method. Hence, according to definition of orbital stability in ref. [41], the order-1 periodic solution $\Gamma^{+}$ is orbitally stable.

For any $G_{0} \in N$, take a numerical sequence $\left\{t_{k}\right\}(k=1,2,3, \ldots)$, where $0<t_{k}<t_{k+1}$ and $\lim _{k \longrightarrow+\infty} t_{k}=$ $+\infty$, such that $\pi\left(t_{k} ; G_{0}, t_{0}\right)=\left((1-p) h, y_{k}\right) \in N$. Due to disjoint of impulsive line, there are two cases on the numerical sequence $\left\{y_{k}\right\}$ when $\tau \in\left(0, \tau_{c}\right)$ and $h<x_{*}:$ : (1) $\left\{y_{k}\right\}$ is a strictly decreasing positive sequence; (2) $\left\{y_{k}\right\}$ is a strictly increasing positive sequence.

For case (1) (see Figure 3(b)), we have $\lim _{k \longrightarrow+\infty} y_{k}=$ $y_{*} \geq y_{C}$ and $y_{k} \geq y_{*}$. Let $Q_{*}=\left((1-p) h, y_{*}\right)$; there exists a point $Q_{\#}=\left((1-p) h, y_{\#}\right)$ when $y_{*}>y_{C}$, where $y_{\#}>y_{*}$, such that point $Q_{*}$ is the order-1 successor point of point $Q_{\#}$. For any point $Q \in \overline{Q_{*} Q_{\#}}$ (i.e., $y_{Q_{*}}<y_{Q}<y_{Q_{\#}}$ ), its order1 successor point is below the point $Q_{*}$. Let point $Q^{+}$be the order-1 successor point of $Q$, then $y_{Q^{+}}<y_{*}$.

Because $\lim _{k \rightarrow+\infty} y_{k}=y_{*}$ and $y_{k} \geq y_{*}$, there exists a positive integer $\Theta_{1}$ such that $y_{Q_{*}}<y_{k}<y_{Q_{\#}}$ for $k>\Theta_{1}$. According to above analysis, there exists a positive integer $\Theta_{2}>\Theta_{1}$ such that $y_{k}<y_{*}$ when $k>\Theta_{2}$, which contradicts with $y_{k} \geq y_{*}$. So, $\lim _{k \longrightarrow+\infty} y_{k}=y_{C}$. Likewise, $\lim _{k \longrightarrow+\infty} y_{k}=$ $y_{C}$ also holds for case (2), which says the order-1 periodic solution $\Gamma^{+}$is orbitally attractive.

Hence, the order-1 periodic solution $\Gamma^{+}$is orbitally asymptotically stable. Since the attraction domain is $\Omega$, the order-1 periodic solution $\Gamma^{+}$is globally orbitally asymptotically stable.

This completes the proof.

Proposition 2. Under condition $(H)$, when $h<x_{*}$ and $\tau>0$, if there exists an order- $k(k \geq 3)$ periodic solution in model 


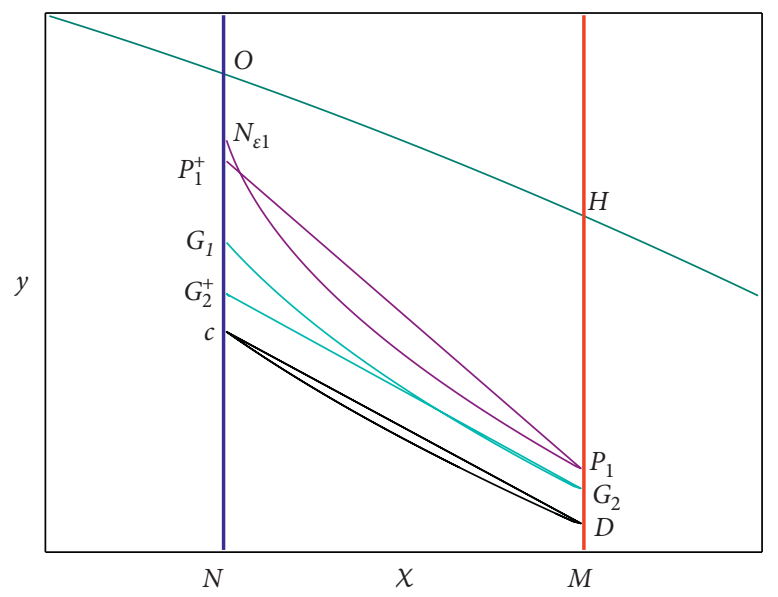

(a)

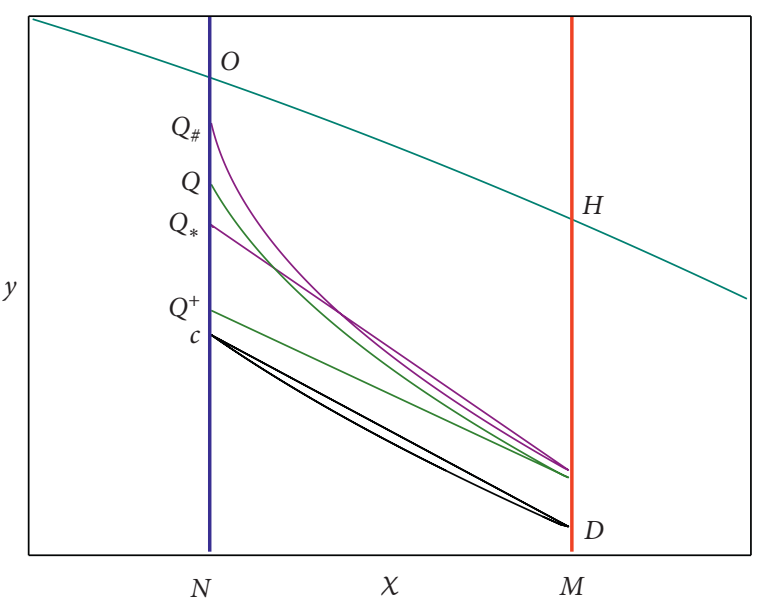

(b)

FIgURE 3: Illustration corresponding to the orbital (a) stability and (b) attractability of order-1 periodic solution in model (2).

(2), setting points $\left((1-p) h, y_{i}\right)(i=1, \ldots, k)$ as the phase points of the order- $k$ periodic solution, then $y_{O} \in\left(\min _{i=1, \ldots, k}\left(y_{i}\right), \max _{i=1, \ldots, k}\left(y_{i}\right)\right)$.

Proof. If $y_{O} \geq \max _{i=1, \ldots, k}\left(y_{i}\right)$, it is obvious that there is no order- $k(k \geq 2)$ according to the proofs in the previous theorems. Hence, we only need to consider $y_{O} \leq \min _{i=1, \ldots, k}\left(y_{i}\right)$. Let points $\left(h, y_{i}^{+}\right)(i=1,2, \ldots, k)$ be the impulsive point of the order- $k$ periodic solution and $y_{i+1}=(1-q) y_{i}^{+}+\tau$; then, $y_{1}=(1-q) y_{k}^{+}+\tau$. Because $y_{O} \leq \min _{i=1, \ldots, k}\left(y_{i}\right)$, for any $l, j \in\{1,2, \ldots, k\}$, if $y_{l}>y_{j}$, then $y_{l}^{+}>y_{j}^{+}$by the disjoint of trajectory of model (1).

For $y_{O} \leq \min _{i=1, \ldots, k}\left(y_{i}\right)$, the relationship among $y_{1}, y_{2}$, and $y_{3}$ is one of the following cases because of the disjoint of the trajectory of model (1) and the disjoint of the impulsive line of model (2):

$$
\begin{aligned}
& \text { (1) } y_{1}>y_{3}>y_{2} \\
& \text { (2) } y_{3}>y_{1}>y_{2} \\
& \text { (3) } y_{2}>y_{1}>y_{3} \\
& \text { (4) } y_{2}>y_{3}>y_{1}
\end{aligned}
$$

For $y_{1}>y_{3}>y_{2}$, we get $y_{1}^{+}<y_{3}^{+}<y_{2}^{+}$, so $y_{2}<y_{4}<y_{3}<y_{1}$ using $y_{i+1}=(1-q) y_{i}^{+}+\tau$. Hence, we claim

$$
y_{2}<\cdots<y_{2 n}<y_{2 n-1}<\cdots<y_{1} \text {. }
$$

When $n=1,2$, it is obvious that inequality (12) holds. Now, suppose inequality (12) holds when $n=\sigma$. In the following, we will prove that inequality (12) also holds when $n=\sigma+1$. Because $y_{2}<\cdots<y_{2 \sigma}<y_{2 \sigma-1}<\cdots<y_{1}$, we have $y_{2}^{+}>\cdots>y_{2 \sigma}^{+}>y_{2 \sigma-1}^{+}>\cdots>y_{1}^{+}$. Consequently, $y_{1}>y_{3}>\cdots>y_{2 \sigma+1}>y_{2 \sigma}>\cdots>y_{2}$, that is, inequality (12) holds when $n=\sigma+1$. Hence, inequality (12) holds when $y_{1}>y_{3}>y_{2}$.

In this case, obviously, for an order- $k$ periodic solution, we have $(1-q) y_{k}^{+}+\tau<y_{1}$ when $y_{1}>y_{3}>y_{2}$, which contradicts to $y_{1}=(1-q) y_{k}^{+}+\tau$. Hence, the order- $k$ periodic solution does not exist. Using similar procedure, it is proved that the order- $k(k \geq 3)$ periodic solution does not exist for cases (2), (3), and (4). So, there is no order- $k(k \geq 3)$ periodic solution in model (2) when $y_{O} \leq \min _{i=1, \ldots, k}\left(y_{i}\right)$. Therefore, $y_{O} \in\left(\min _{i=1, \ldots, k}\left(y_{i}\right), \max _{i=1, \ldots, k}\left(y_{i}\right)\right)$ when an order$k(k \geq 3)$ periodic solution exists in model (2).

This completes the proof.

By Proposition 2, the following theorem will exist.

Theorem 4. Under condition (H), if $h<x_{*}$ and $\tau \geq y_{O}$, then there exists a globally orbitally asymptotically stable and unique order-1 periodic solution or a unique order-1 periodic solution and an order-2 solution coexists in model (2) for any $q \in(0,1)$, and there is no order- $k(k \geq 3)$ periodic solution.

Proof. By Proposition 2, it is obvious that there is no order$k(k \geq 3)$ periodic solution in model (2) because $(1-q) y+$ $\tau>\tau \geq y_{O}$ for any $y>0$. If order- 2 periodic solution does not exist, then a globally orbitally asymptotically stable and unique order-1 periodic solution exists using Theorems 2 and 3 . If an order-2 periodic solution exists, then the order-2 periodic solution coexists with a unique order-1 periodic solution by Theorem 2 .

This completes the proof.

Theorem 5. Under condition (H), if $h<x_{*}$, for given $\tau>0$, then all the non-negative solutions of model (2) which start in $\Omega$ are ultimately bounded, and the bound depends on parameter $\tau, q, p$, and $h$.

Proof. By model (2) and the space $\Omega$, obviously, $0<\lim _{t \longrightarrow+\infty} \sup x(t) \leq h$ and $0 \leq \lim _{t \longrightarrow+\infty} \sup y(t)$ hold. In addition, set $\Lambda_{N}=\{y \mid y$ is the ordinate of phase point $\}$ and $\Lambda_{M}=\{y \mid y$ is the ordinate of impulsive point $\}$. Then, $y_{O_{1}}=\max \left(\Lambda_{M}\right)$, so we can obtain $y_{O_{1}^{+}}=\max \left(\Lambda_{N}\right)$ by $y_{O_{1}^{+}}=(1-q) y_{O_{1}}+\tau$. Hence, $\quad \lim _{t \longrightarrow+\infty} \sup y(t) \leq y_{O_{1}^{+}}$, which obviously depends on $\tau, q, p$, and $h$.

This completes the proof.

From Theorem 5, it is not difficult to find that there is a rough estimate of the bound, and we have the following remark. 
Remark 1. Under condition $(H)$, if $h<x_{*}$, then $0<\lim _{t \rightarrow+\infty} \sup x(t) \leq h$ $0 \leq \lim _{t \longrightarrow+\infty} \sup y(t)<(1-q) y_{H}+\tau$.

Proposition 3. Under condition $(H)$, if there exists a point $S$ such that $F_{k}(S)=0$ in model (2) when $h<x_{*}$, then there exists an order- $k_{f}$ periodic solution, where the positive integer $k_{f}$ is a factor of the positive integer $k$, including 1 and $k$.

Proof. Suppose positive integer $k_{f}$ is not a factor of the positive integer $k$, then let $k_{r}$ be the remainder of $k / k_{f}$ and $k_{q}$ be the quotient of $k / k_{f}$. Because there exists an order- $k_{f}$ periodic solution in model (2), without loss of generality, assume $F_{k_{f}}(S)=0$. Obviously, we have $F_{k_{q} k_{f}}(S)=0$, but $F_{k_{r}}(S) \neq 0$. Hence, the equation $F_{k_{q} k_{f}+k_{r}}(S) \neq 0$, which is a contradiction with $F_{k}(S)=0$ because $k=k_{q} k_{f}+k_{r}$. So, the positive integer $k_{f}$ is a factor of the positive integer $k$. When the positive integer $k_{f}$ is a factor of the positive integer $k$, $F_{k}(S)=0$ is obvious if there exists an order- $k_{f}$ periodic solution in model (2).

This completes the proof.

Proposition 4. Under condition $(H)$, if there exists a point $\mathrm{O}_{0}$ below point $\mathrm{O}$ such that $\mathrm{F}_{3}\left(\mathrm{O}_{0}\right)<0$ holds when $h<x_{*}$, $0<\tau<y_{O}, p \in(0,1)$, and $q \in(0,1)$, then model (2) has an order-3 periodic solution when $F_{1}(O)>0$, and the order-3 periodic solution coexists with an order-1 periodic solution.

Proof. Setting $E=N \cap\{(x, y) \mid 0<x \leq h, y=0\}$, we have $F_{1}(E)>0, F_{2}(E)>0$ and $F_{3}(E)>0$. Hence, there exists a point $O_{*} \in \overline{E O_{0}}$ such that $F_{3}\left(O_{*}\right)=0$ because $F_{3}\left(O_{0}\right)<0$, so an order- $k(k=1$ or 3$)$ periodic solution exists by Proposition 3. However, owing to $F_{1}(O)>0$, it is obvious that there is no order- 1 periodic solution in space $\Pi$ by the proof of Theorem 2. Thus, the periodic solution is the order-3 one. Furthermore, because $F_{1}(O)>0$, there exists an order-1 periodic solution according to the proof of Theorem 2, and its phased point is above point $O$. Therefore, the order-3 periodic solution coexists with an order-1 periodic solution.

This completes the proof.

Although it is not proved that point $O_{0}$ must exist in Proposition 4, it provides a method to search an order-3 periodic solution for numerical analysis. Furthermore, the existence of order-3 periodic solution means that order$k(k>3)$ periodic solution and chaos exist in model (2) by Sarkovskii's theorem [42] and $\mathrm{Li}$ and Yorke's theorem [43].

\section{Numerical Results}

In the previous section, we have analyzed the properties of periodic solution in model (2), including the existence, uniqueness, stability, and boundedness. In this section, we will further show the complex dynamics induced by impulsive control using the numerical simulations. Since the focus of this paper is the role of impulsive control in the population dynamics, the parameters of model (1) are fixed: $r=0.9, K=100, b=1.2, a=7, \alpha=0.7$, and $m=0.7$, and we can obtain the equilibrium $E_{*}=(35,20.475)$ with index +1 .
Obviously, condition $(H)$ always holds under this parameter set.

By Theorem 1, there only exists a semitrivial periodic solution in model (2) when $\tau=0$ and $h<x_{*}$. Given $h=3, p=0.6, q=0.5$, and $\tau=0$, we get a semitrivial periodic solution as shown in Figure 4(a), and its period is about 1.309. In addition, we also obtain the theoretical value of the period by $T=(1 / r) \ln (K-(1-p) h /$ $(1-p)(K-h))$, which is the same as the numerical one. When $h=3$ and $p=0.6$, the point $O$ is fixed at $(1.2,6.0762)$. The numerical simulation shows that all the successor points of any trajectory except the semitrivial solution are below initial point in model (2) and these trajectories converge towards the semitrivial periodic solution (see Figure 4(b)).

When $\tau>0$, however, the semitrivial periodic solution disappears. Then, an order-1 periodic solution emerges and it should be unique by Theorem 2 . In phase set $N$, because the order-1 successor point of any point above point $O$ is below point $O_{1}^{+}$, we compute the order- 1 successor function $F_{1}(y)$ where $y \in\left(0, y_{\mathrm{O}_{1}^{+}}\right]$for different values of $\tau$ (see Figure 5(a)), where $h=32, p=0.1$, and $q=0.1$. In the rest of this section, we will employ this control parameter set unless otherwise specified.

From Figure 5(a), it is not difficult to find that the equation $F_{1}(y)=0$ has a unique root, which means that there exists a unique order-1 periodic solution. Hence, we carry out some numerical simulations with respect to these roots of $F_{1}(y)=0$, and the results verify that these solutions are order-1 periodic solutions as shown in Figure 5(b). But we find that some of these order-1 periodic solutions are unstable, which indicates that there may exist order- $k$ periodic solution for some values of $\tau$.

In order to illustrate the existence of order- $k$ periodic solution, we further compute order-1 successor function, order-2 successor function, and order-3 successor function, and the results are very interesting and are shown in Figure 6(a). From Figure 6(a), it is easy to find that the equation $F_{1}(y)=0$ has a unique root, the equation $F_{2}(y)=$ 0 has three roots, and the equation $F_{3}(y)=0$ has seven roots. Furthermore, we can find that the root of $F_{1}(y)=0$ is the common root of three equations: $F_{1}(y)=0, F_{2}(y)=0$, and $F_{3}(y)=0$, which means that there exists an order-1 periodic solution corresponding to this root. Except this root, the equation $F_{2}(y)=0$ still has two roots which indicate an order- 2 periodic solution exists in virtue of the uniqueness of order-1 periodic solution. For order-3 successor function $F_{3}(y)$, the equation $F_{3}(y)=0$ has six roots except for the root of $F_{1}(y)=0$.

Except for the root of $F_{1}(y)=0$, it is not difficult to find that $F_{1}(y)$ and $F_{2}(y)$ are positive or negative definite in a small neighborhood of each root of $F_{3}(y)=0$, which means that there may exist two order-3 periodic solutions in model (2). Thus, we perform some numerical simulations to show the solutions corresponding to these roots. The results show that a stable order-3 periodic solution coexists with an unstable order-3 periodic solution, an unstable order-2 periodic solution, and an unstable order-1 periodic solution (see Figure 6(b)). Additionally, the numerical solutions for 


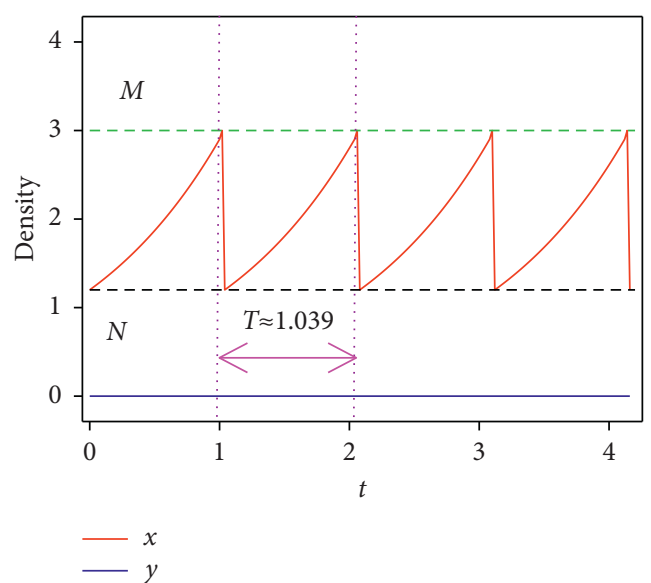

(a)

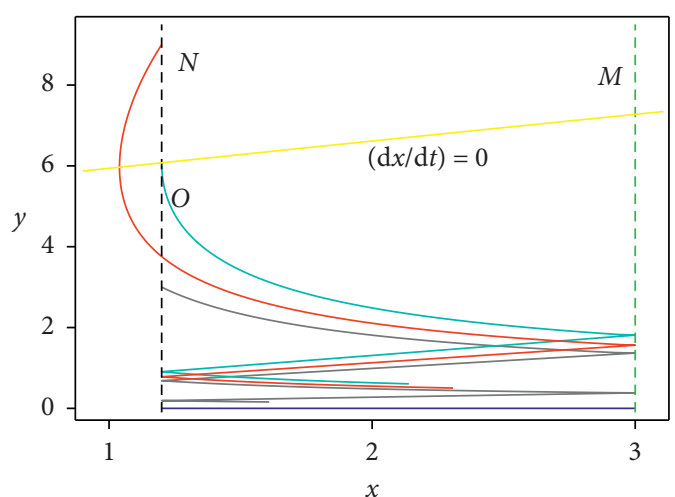

(b)

Figure 4: (a) The semitrivial periodic solution of model (2) (the red curve denotes the density of population $x$ and the blue line denotes the density of population $y$ ). (b) The proof of trajectory whose successor points are below initial points.

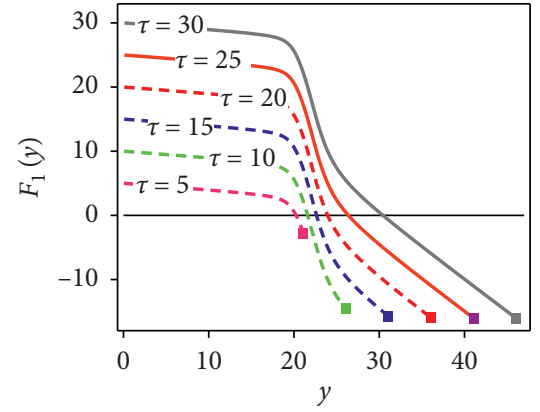

(a)

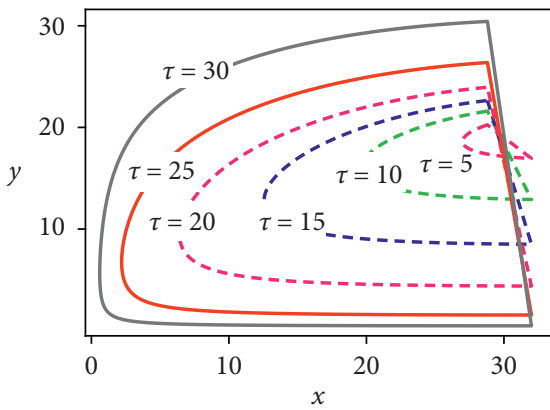

(b)

Figure 5: (a) The order-1 successor function $F_{1}(y)$ for $y \in\left(0, y_{S}\right)$, where the solid square denotes the value of $y_{S}$. (b) Order-1 periodic solutions corresponding to roots of $F_{1}(y)=0$, where the dashed curves denote the unstable order-1 periodic solutions and the solid curves denote the stable ones.

prey $y$ are given to further exhibit these periodic solutions, as shown in Figure 6(c).

The above results indicate that the order-1 periodic solutions are not always stable. Using order- 1 and order- 2 successor functions, we compute $y$-value with respect to the phased point of order- 1 periodic solution to simulate its variation as parameter $\tau$ increases (see Figure 7(a)). From Figure $7(\mathrm{a})$, the order-1 periodic solution is stable before parameter $\tau$ reaches line $l_{1}$, and the order-1 periodic solution is still stable near $l_{1}$ when parameter $\tau$ is beyond $l_{1}$. This result is in accord with Theorem 3. Keeping parameter $\tau$ increasing, the order-1 periodic solution loses its stability until it is beyond $l_{3}$. It is well worth noting that model (2) only has order-1 and order- 2 periodic solution when parameter $\tau$ is between $l_{2}$ and $l_{3}$, which agrees with Theorem 4 . Furthermore, Figure 7 (a) demonstrates that the order-1 periodic solution is unique, which supports the result in Theorem 2. Additionally, Figure 7 (a) also shows that $y=$ $y(\tau)$ is a monotonously increasing function with respect to $\tau$, which is consistent with Proposition 1 .

In addition, our numerical results also indicate that the phased point of order-1 periodic solution rises along phase set as parameter $\tau$ increases. In order to present the stability of order-1 periodic solution, we compute two groups of order- $k$ successor points whose initial point is on the sides of the phased point of order-1 periodic solution, respectively. It is revealed that these points monotonously converge towards the phased point of order- 1 periodic solution (see Figure 7(b)). Hence, this order-1 periodic solution is stable due to the nonexistence of other periodic solutions.

In order to display complex dynamics, we chose parameter $\tau$ as the controlled parameter and ran numerous simulations using a wide range of parameter $\tau$ to show the bifurcation diagram (see Figure 8(a)). Since the stability of order-1 periodic solution has been discussed above (Figure 7(a)), we will present the dynamics when the order-1 periodic solution is unstable. From Figure 8(a), increased parameter $\tau$ can generate chaos by a cascade of perioddoubling bifurcation. Keeping parameter $\tau$ increasing, an order-3 periodic solution bifurcates from chaos. Subsequently, chaos occurs again with the increase of parameter $\tau$. Finally, chaos disappears again via a cascade of inverse period-doubling bifurcation at higher values in the 


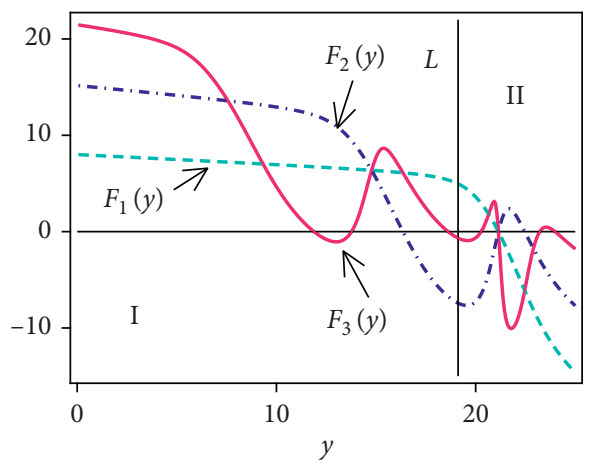

(a)

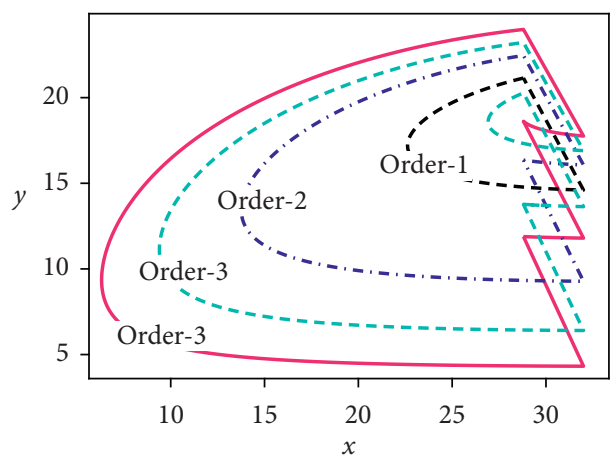

(b)

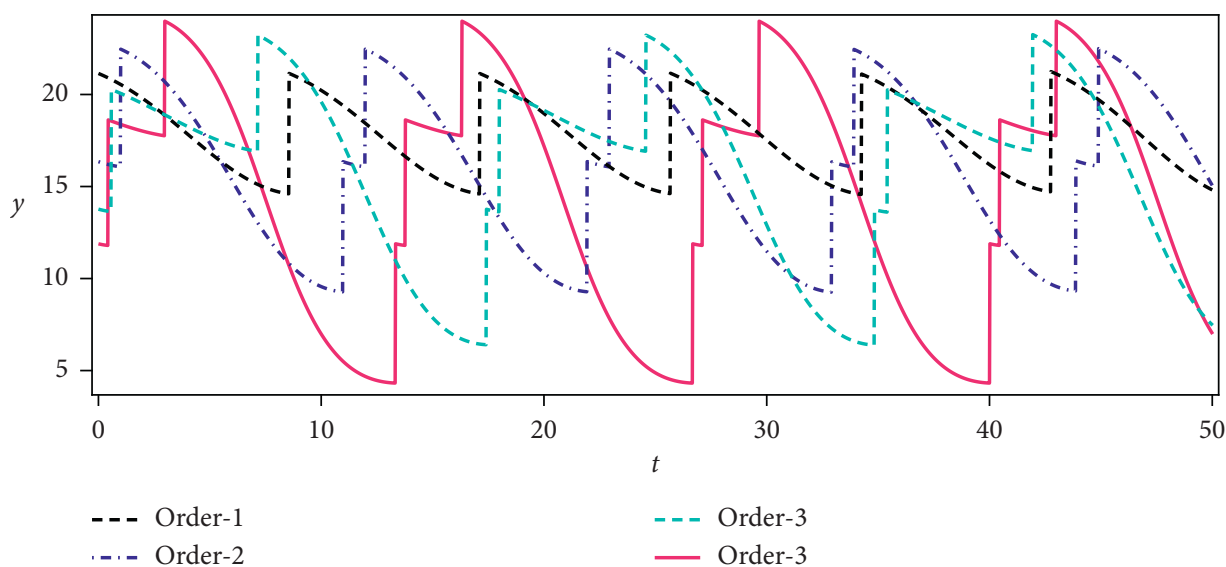

(c)

Figure 6: (a) The order-1 successor function $F_{1}(y)$, the order-2 successor function $F_{2}(y)$, and the order-3 successor function $F_{3}(y)$, where the line $L$ denotes line $y=y_{O}$. (b) An order-1 periodic solution corresponding to the root of $F_{1}(y)=0$, an order-2 periodic solution corresponding to roots of $F_{2}(y)=0$, and two order-3 periodic solutions corresponding to roots of $F_{3}(y)=0$, where the dashed curves denote the unstable periodic solutions and the solid curves denote the stable ones. (c) Time-series of population $y$ corresponding to the periodic solutions in (b), where $\tau=8$.

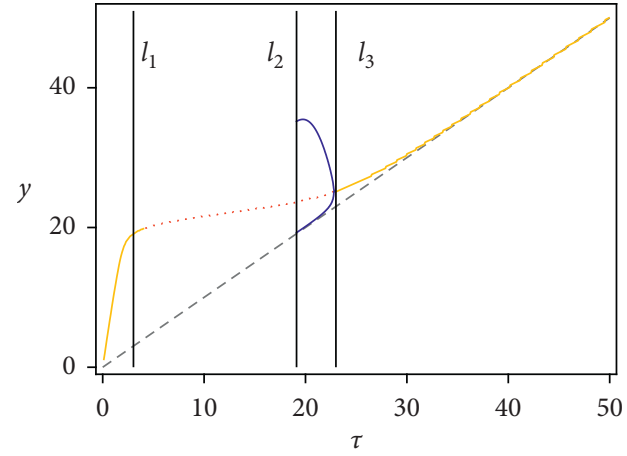

(a)

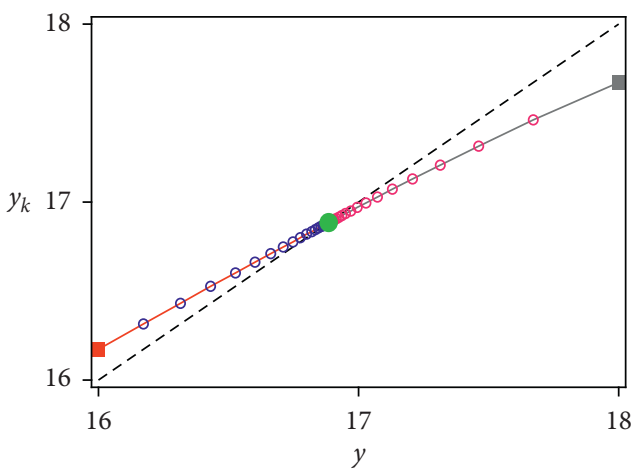

(b)

FIGURE 7: (a) The existence and stability of the order-1 periodic solution (the solid yellow curve represents stability of the order-1 periodic solution, the dashed red curve represents instability of the order-1 periodic solution, and the solid blue curve represents the stable order-2 periodic solution; the line $l_{1}$ denotes $\tau=y_{S}-(1-q) \pi\left(t_{1} ; O, t_{0}\right)$, where $t_{1}$ is the moment when the trajectory $\pi\left(t_{1} ; O, t_{0}\right)$ first met line $x=h$, the line $l_{2}$ denotes $\tau=y_{O}$, and the line $l_{3}$ denotes the critical value of parameter $\tau$ where the order-1 periodic solution obtain its stability again). (b) The proof of stability of the order-1 periodic solution (the solid squares represent initial points, the lines with circles denote the successor points, and the solid green circle denotes the phase point of order-1 periodic solution).

simulated range of parameter $\tau$. To confirm the occurrence of chaos further, we calculate the largest Lyapunov exponents in the same range of parameter $\tau$ as Figure 8(a), which agrees with the bifurcation diagram (see Figure 8(b)). In addition, dozens of time-series of population $y$ for differently initial values are given in Figure 8(c) to further show 


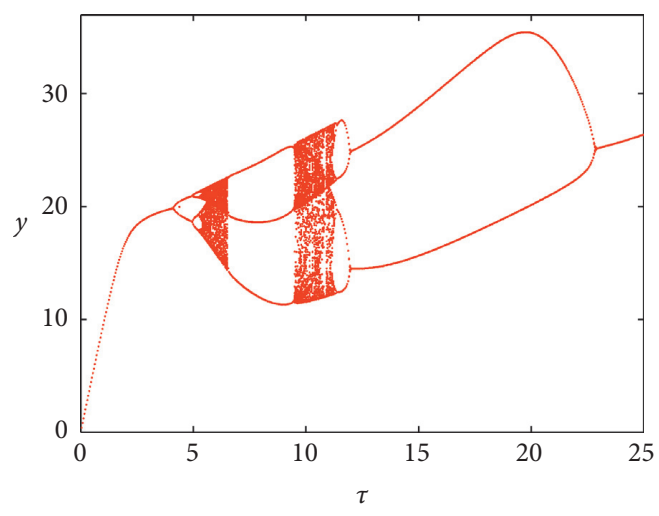

(a)

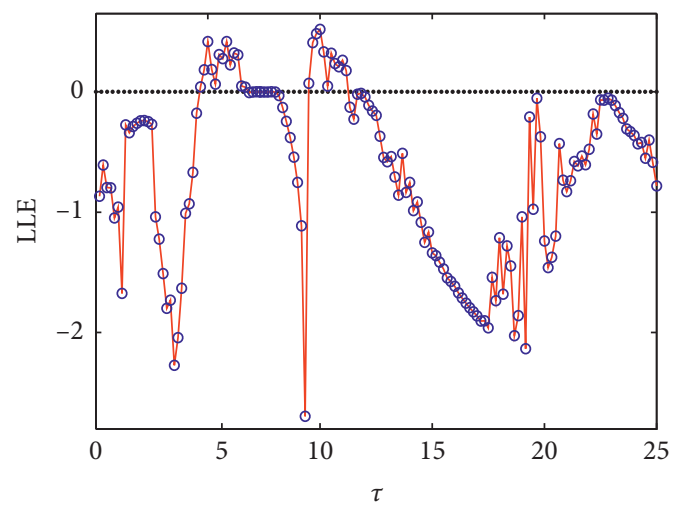

(b)

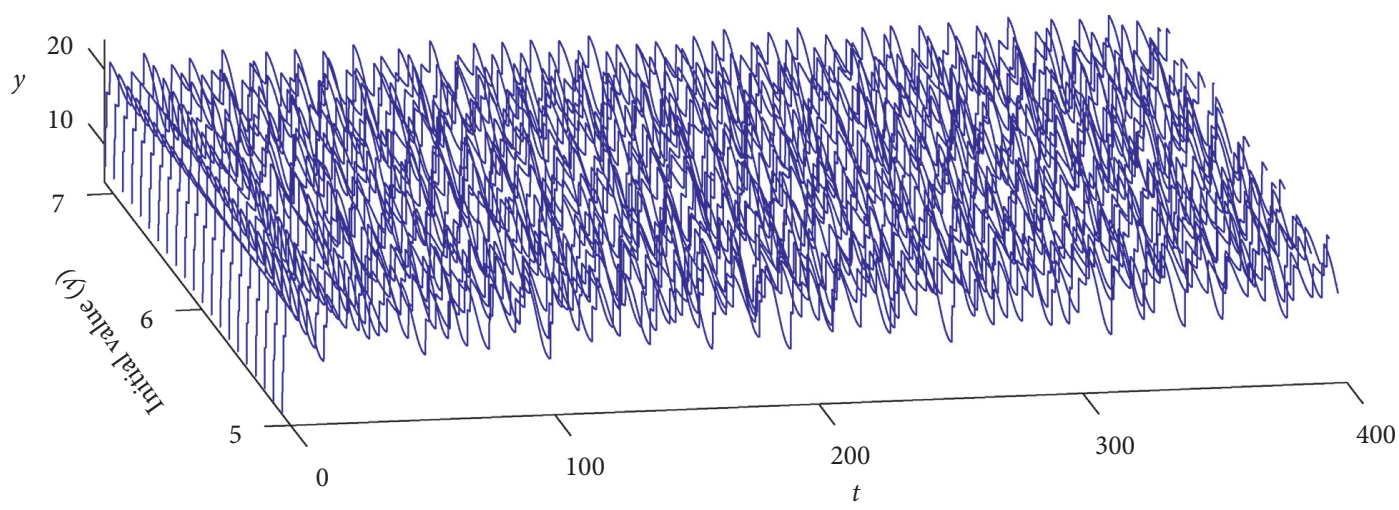

(c)

Figure 8: (a) The bifurcation diagram with respect to parameter $\tau$ for model (2). (b) The variation of the Lyapunov exponents with respect to the parameter $\tau$, where the symbol "LLE" denotes the largest Lyapunov exponent. (c) Time-series of population y with different initial values for $\tau=6$.

chaos, which suggests that all states described by these timeseries are in disorder.

\section{Conclusion}

In this paper, we proposed an ecological model with statedependent impulsive control strategy to investigate the effect of impulsive control on prey-predator dynamics. Theoretical results suggest that model (2) has a globally orbitally asymptotically stable semitrivial periodic solution when parameter $\tau=0$ and $h<x_{*}$, where condition $(H)$ holds to guarantee existence of positive equilibrium $E_{*}$. When $\tau>0$, the semitrivial periodic solution disappears, but the theoretical analysis shows that a unique order- 1 periodic solution emerges for any $q \in(0,1)$ and $p \in(0,1)$.

Furthermore, we find that the order-1 periodic solution is globally orbitally asymptotically stable when the value of $\tau$ is below $\tau_{c}$. Additionally, when the value of $\tau$ is beyond $y_{o}$, theoretical analysis reveals that a globally orbitally asymptotically stable order-1 periodic solution exists or coexistence of a unique order- 1 periodic solution and order- 2 periodic solution occurs.

Additionally, the existence of order- $k$ is theoretically analyzed based on equation $F_{k}(y)=0$, which demonstrates that an order- $k_{f}$ periodic solution exists when $F_{k}(y)=0$, and $k_{f}$ is a factor of the positive integer $k$, including 1 and $k$.
According to this result, we discuss the existence of order-3 periodic solution. Although we cannot get the exact range of parameter $\tau$ with respect to the existence of order-3 periodic solution, our results imply that the order- 3 periodic solution may exist for $\tau \in\left(\tau_{c}, y_{O}\right)$, which even means that chaos may occur.

In order to study dynamics induced by impulsive control further, a series of numerical simulations are carried out, which agreed with the theoretical results well. Numerical simulations show that order- 1 , order- 2 , and order- 3 periodic solutions can coexist. Specially, we find the coexistence of double order-3 periodic solutions. Moreover, numerical bifurcation analysis shows that an unstable order-1 periodic solution and a stable order- 2 periodic solution can coexist for $\tau>y_{o}$, but there exists only a stable order-1 periodic solution when $\tau$ is beyond some critical value. In addition, numerical simulations show that chaos occurs. Obviously, dynamics induced by impulsive control is much richer.

The theoretical and numerical results predict that the population $x$ can be controlled using the state-dependent impulsive control strategies. Because $h<x_{*}$, the method by impulsive harvesting population $x$ was a unique choice which can keep the density of population $x$ below the critical value. Our results shows that the control period is much lower when the released amount $\tau$ is much smaller, which means that the control frequency is much higher. But from 
the viewpoint of management for some ecological problems (e.g., pest outbreak, phytoplankton blooms, etc.), it is always hoped that the control period is much longer. Thus, much more predators are needed to be released. However, our results also indicate that chaos may occur for some values of $\tau$, which is unfavourable for prediction of control period. Hence, the released amount of predator should be chosen carefully. Obviously, our results are much more interesting, and we expect that these results are helpful to the management of ecological problems.

\section{Data Availability}

The data used to support the findings of this study are included within the article.

\section{Conflicts of Interest}

The author declares that there are no conflicts of interest.

\section{Acknowledgments}

This study was supported by the Zhejiang Provincial Natural Science Foundation of China (grant no. LQ18C030002) and the National Natural Science Foundation of China (grant no. 61901303).

\section{References}

[1] A. Dobson, "Mathematical models for emerging disease," Science, vol. 346, pp. 1294-1295, 2014.

[2] G. A. Jackson, "Phytoplankton growth and zooplankton grazing in oligotrophic oceans," Nature, vol. 284, pp. 439-441, 1980.

[3] J. Huisman, T. Pham, N. Nga, D. M. Karl, and B. Sommeijer, "Reduced mixing generates oscillations and chaos in the oceanic deep chlorophyll maximum," Nature, vol. 439, pp. 322-325, 2006.

[4] C. J. Dai, H. G. Yu, Q. Guo et al., "Dynamics induced by delay in a nutrient-phytoplankton model with multiple delays," Complexity, vol. 2019, Article ID 3879626, 16 pages, 2019.

[5] V. Lakshmikantham, D. D. Bainov, and P. S. Simeonov, Theory of Impulsive Differential Equations, World Scientific, Singapore, 1989.

[6] D. D. Bainov and P. S. Simeonov, Systems with Impulsive Effect: Stability, Theory and Applications, Wiley, New York, NY, USA, 1989.

[7] E. M. Bonotto and M. Federson, "Limit sets and the Poincaré Bendixson theorem in impulsive semidynamical systems," Journal of Differential Equations, vol. 224, no. 9, pp. 23342349, 2008.

[8] J. J. Nieto and D. O’Regan, "Variational approach to impulsive differential equations," Nonlinear Analysis: Real World Applications, vol. 10, no. 2, pp. 680-690, 2009.

[9] R. P. Agarwal, A. S. Awan, D. O’Regan, and A. Younus, "Linear impulsive Volterra integro-dynamic system on time scales," Advances in Difference Equations, vol. 2014, Article ID 6, 2014.

[10] A. J. Terry, "Biocontrol in an impulsive predator-prey model," Mathematical Biosciences, vol. 256, pp. 102-115, 2014.

[11] X. D. Li, M. Bohner, and C. K. Wang, "Impulsive differential equations: periodic solutions and applications," Automatica, vol. 52, pp. 173-178, 2015.
[12] J. Yang, Y. S. Tan, and R. A. Cheke, "Modelling effects of a chemotherapeutic dose response on a stochastic tumourimmune model," Chaos, Solitons \& Fractals, vol. 123, pp. 1-13, 2019.

[13] S. Y. Tang and R. A. Cheke, "State-dependent impulsive models of integrated pest management (IPM) strategies and their dynamic consequences," Journal of Mathematical Biology, vol. 50, no. 3, pp. 257-292, 2005.

[14] J. Yang and Y. S. Tan, "Effects of pesticide dose on Holling II predator-prey model with feedback control," Journal of Biological Dynamics, vol. 12, no. 1, pp. 527-550, 2018.

[15] G. R. Jiang, Q. S. Lu, and L. N. Qian, "Complex dynamics of a Holling type II prey- predator system with state feedback control," Chaos, Solitons \& Fractals, vol. 31, pp. 448-461, 2007.

[16] C. J. Dai and M. Zhao, "Mathematical and dynamic analysis of a prey-predator model in the presence of alternative prey with impulsive state feedback control," Discrete Dynamics in $\mathrm{Na}$ ture and Society, vol. 2012, Article ID 724014, 19 pages, 2012.

[17] C. J. Dai, M. Zhao, and L. S. Chen, "Dynamic complexity of an Ivlev-type prey-predator system with impulsive state feedback control," Journal of Applied Mathematics, vol. 2012, Article ID 534276, 17 pages, 2012.

[18] L. F. Nie, J. G. Peng, Z. D. Teng, and L. Hu, "Existence and stability of periodic solution of a Lotka-Volterra predatorprey model with state dependent impulsive effects," Journal of Computational and Applied Mathematics, vol. 224, no. 2, pp. 544-555, 2009.

[19] K. B. Sun, T. H. Zhang, and Y. Tian, "Dynamics analysis and control optimization of a pest management predator-prey model with an integrated control strategy," Applied Mathematics and Computation, vol. 292, pp. 253-271, 2017.

[20] G. P. Pang and L. S. Chen, "Periodic solution of the system with impulsive state feedback control," Nonlinear Dynamics, vol. 78, no. 1, pp. 743-753, 2014.

[21] S. Y. Tang, B. Tang, A. L. Wang, and Y. N. Xiao, "Holling II predator-prey impulsive semi-dynamic model with complex Poincaré map," Nonlinear Dynamics, vol. 81, no. 3, pp. 1575-1596, 2015.

[22] J. Yang, Y. S. Tan, and R. A. Cheke, "Complex dynamics of an impulsive Chemostat model," International Journal of Bifurcation and Chaos, vol. 29, no. 8, Article ID 1950101, 2019.

[23] D. Z. Li, H. D. Cheng, and Y. Liu, "Dynamic analysis of Beddington-DeAngelis predator-prey system with nonlinear impulse feedback control," Complexity, vol. 2019, Article ID 5308014, 13 pages, 2019.

[24] P. S. Simeonov and D. D. Bainov, "Orbital stability of periodic solutions of autonomous systems with impulse effect," International Journal of Systems Science, vol. 19, no. 12, pp. 2561-2585, 1988.

[25] L. N. Qian, Q. S. Lu, Q. G. Meng, and Z. S. Feng, "Dynamical behaviors of a prey-predator system with impulsive control," Journal of Mathematical Analysis and Applications, vol. 363, no. 1, pp. 345-356, 2010.

[26] L. S. Chen, "Pest control and geometric theory of semi-dynamical systems," Journal of Beihua University, vol. 12, pp. 1-9, 2011.

[27] C. J. Dai, M. Zhao, and L. S. Chen, "Homoclinic bifurcation in semi-continuous dynamic systems," International Journal of Biomathematics, vol. 5, no. 6, Article ID 1250059, 2012.

[28] H. J. Guo, X. Y. Song, and L. S. Chen, "Qualitative analysis of a Korean pine forest model with impulsive thinning measure," Applied Mathematics and Computation, vol. 234, pp. 203-213, 2014. 
[29] X. H. Ji, S. L. Yuan, and L. S. Chen, "A pest control model with state-dependent impulses," International Journal of Biomathematics, vol. 8, no. 1, Article ID 1550009, 2015.

[30] Y. Tian, K. B. Sun, and L. S. Chen, "Geometric approach to the stability analysis of the periodic solution in a semi-continuous dynamic system," International Journal of Biomathematics, vol. 7, no. 2, Article ID 1450018, 2014.

[31] T. Zhang, X. Meng, R. Liu, and T. Zhang, "Periodic solution of a pest management Gompertz model with impulsive state feedback control," Nonlinear Dynamics, vol. 78, no. 2, pp. 921-938, 2014.

[32] M. Z. Huang and X. Y. Song, "Periodic solutions and homoclinic bifurcations of two predator-prey systems with nonmonotonic functional response and impulsive harvesting," Journal of Applied Mathematics, vol. 2014, Article ID 803764, 11 pages, 2014.

[33] Q. Z. Xiao, B. X. Dai, B. X. Xu, and L. S. Bao, "Homoclinic bifurcation for a general state-dependent Kolmogorov type predator-prey model with harvesting," Nonlinear Analysis: Real World Applications, vol. 26, pp. 263-273, 2015.

[34] C. J. Wei and L. S. Chen, "Periodic solution and heteroclinic bifurcation in a predator- prey system with Allee effect and impulsive harvesting," Nonlinear Dynamics, vol. 76, no. 2, pp. 1109-1117, 2014.

[35] M. Z. Huang, S. Z. Liu, X. Y. Song, and L. S. Chen, "Dynamics of unilateral and Bilateral control systems with state feedback for renewable resource management," Complexity, vol. 2020, Article ID 9453941, 16 pages, 2020.

[36] Z. Z. Shi, J. M. Wang, Q. J. Li, and H. D. Cheng, "Control optimization and homoclinic bifurcation of a prey-predator model with ratio-dependent," Advances in Difference Equations, vol. 2019, no. 1, 2019.

[37] B. Liu and Y. Tian, "Dynamics on a Holling II predator-prey model with state-dependent impulsive control," International Journal of Biomathematics, vol. 5, no. 3, Article ID 1260006, 2012.

[38] G. P. Pang, Z. Q. Liang, W. J. Xu, L. J. Li, and G. Fu, “A pest management model with stage structure and impulsive state feedback control," Discrete Dynamics in Nature and Society, vol. 2015, Article ID 617379, , 2015.

[39] T. Yoshida, L. E. Jones, S. P. Ellner, G. F. Fussmann, and N. G. Hairston Jr., "Rapid evolution drives ecological dynamics in a predator-prey system," Nature, vol. 424, no. 6946, pp. 303-306, 2003.

[40] G. F. Fussmann, S. P. Ellner, K. W. Shertzer, and N. G. Hairston Jr., "Crossing the Hopf bifurcation in a live predator-prey system," Science, vol. 290, pp. 1358-1360, 2000.

[41] D. W. Jordan and P. Smith, Nonlinear Ordinary Differential Equations: An Introduction for Scientists and Enginerrs, Oxford University, New York, NY, USA, 4th edition, 2007.

[42] R. L. Devaney, An Introduction to Chaotic Dynamical Systems, Westview Press, Bounlder, CO, USA, 2003.

[43] T. Y. Li and J. A. Yorke, "Period three implies chaos," The American Mathematical Monthly, vol. 82, pp. 985-992, 1975. 